\title{
Potential Viability of a Fast-Acting Micro-Solenoid Valve for Pulsed Detonation Fuel Injection
}

\author{
F.K. Lu* and D.S. Jensen† \\ University of Texas at Arlington, Arlington, Texas 76019
}

\begin{abstract}
The potential for fast-acting solenoid valves to meet the demands of pulsed detonation fuel injection and other high-frequency devices is presented. The micro-valve was found to performance well above the manufacturer's rated frequency under no-load conditions, indicating the ability of springreturn micro-valves to function reliably at high frequencies. An examination of the electromagnetic force computed from the experimental displacement response demonstrates that flux saturation occurs early in the rise time. Opening and closing phases are examined and compared under ambient pressure conditions.
\end{abstract}

\section{Nomenclature}

$A_{x} \quad$ cross sectional variable air gap normal to magnetic flux path $\left(\mathrm{m}^{2}\right)$

$C_{d} \quad$ damping coefficient $(\mathrm{kg} / \mathrm{s})$

$F_{e} \quad$ electromagnetic force of attraction (N)

$F_{g} \quad$ gas pressure force exerted on the core in the $x$ direction $(\mathrm{N})$

$i_{e} \quad$ excitation current (A)

$i_{m} \quad$ magnetization current (A)

$k \quad$ spring constant $\left(\mathrm{kg} / \mathrm{s}^{2}\right)$

$L_{l} \quad$ flux leakage inductance $(\mathrm{H})$

$L(\phi, x) \quad$ non-linear coil inductance $(\mathrm{H})$

$m$

$n$

$x$
$N$

$R_{c}$

$r_{c}$

$T$

$V_{s}$

$V_{\phi}$

$W$

$\Re$

$\Re_{\mathrm{m}}$

$\delta$

$\mu_{o}$

mass of movable valve core and spring assembly $(\mathrm{kg})$

integer number of successive (positive) peaks

(Fig. 3)

number of coil turns

variable core loss resistance $(\Omega)$

actual coil resistance $(\Omega)$

period of oscillation (s)

supply voltage $(\mathrm{V})$

flux leakage voltage (V)

energy stored in coil $(\mathrm{J})$

variable air gap length in the direction of the

magnetic flux path $(\mathrm{m})$

total reluctance $(\mathrm{A} / \mathrm{Wb})$

core reluctance $(\mathrm{A} / \mathrm{Wb})$

logarithmic decrement

permeability of free space $\left(4 \pi \times 10^{-7}\right.$

$\mathrm{Wb} / \mathrm{A} \cdot \mathrm{m})$

*Professor, Mechanical and Aerospace Engineering Department, and Director, Aerodynamics Research Center, Box 19018, Arlington, TX 76019. Associate Fellow AIAA.

$\dagger$ Research Assistant, Aerodynamics Research Center, Box 19018, Arlington, TX 76019. Student Member AIAA.

Copyright (C) 2003 by F.K. Lu and D.S. Jensen. Published by the American Institute of Aeronautics and Astronautics, Inc. with permission.

$\begin{array}{ll}\phi & \text { magnetic flux }(\mathrm{Wb}) \\ \omega_{\mathrm{d}} & \text { damped natural frequency }(\mathrm{rad} / \mathrm{s}) \\ \omega_{\mathrm{n}} & \text { undamped natural frequency }(\mathrm{rad} / \mathrm{s}) \\ \zeta & \text { damping ratio }\end{array}$

\section{Introduction}

$\mathcal{T}$ HERE is consensus, based on systems studies and on the order of at least $100 \mathrm{~Hz}$ are needed for aerospace propulsion systems. While there are various valveless concepts, they remain on the drawing boards. Practical demonstrators of pulse detonation technology tend to use mechanical, valve assemblies ${ }^{1,2}$ or solenoid valves for delivering the fuel, oxidizer and purge air. Most of these valves operate at frequencies lower than the theoretical requirements, partly due to limitations in valve performance. However, as cycling frequencies increase, less time is available to inject fuel. In many cases of gaseous fuel injection, the valve is already choked. Thus the injection orifice must be enlarged in order to deliver the required mass flow in the shorter available time and the fuel must be injected at higher pressure. Unfortunately, this equates to a slower response for typical rotary-type valves and hinders the system's ability to deliver the appropriate gases under stringent timing conditions. Further, in terms of overall engine design, weight and maintenance considerations tend not to favor these mechanical systems.

The possibility of utilizing high-frequency solenoid valves, with the advantages of electronic control, appears to be a viable solution for overcoming the limitations inherent in mechanical valves. These valves, with life spans of hundreds of millions of cycles, may be viable for missile and rocket applications. Solenoid valves are also made of materials compatible with the fluids used in pulse detonation engines. A search of the open literature reveals that solenoid valves used in pulse detonation ap- 
plications operate at low frequencies, typically 10-20 $\mathrm{Hz}$.

To operate solenoid valves at the high frequencies of interest, valve behavior under high-frequency excitation must be understood. For example, a typical assumption of the fuel injection period is 30 percent of the cycle time. ${ }^{3}$ For $100 \mathrm{~Hz}$ operation, a satisfactory injection time might be $3 \mathrm{~ms}$. Allowing a 10 percent deviation from an instantaneous response, an acceptable valve response would then be $0.3 \mathrm{~ms}$.

Solenoid valve manufacturers usually do not provide information on response time or frequency response. Further, it is only recently that the dynamic behavior of solenoid valves has received comprehensive treatment, and few definitive works have been published. ${ }^{4-7}$ Hong $^{6}$ developed a robust analytical model which can be adapted to most solenoid valve geometries. The model included the effects of core losses and flux leakage However, experimental data and some parameter estimation are still necessary to completely characterize a valve's response. Losses are best determined by experimentally measuring the solenoid force directly., ${ }^{5,6}$ Performance, however, can be examined through the relatively simple measurement of the displacement response. Such a method is presented in the following sections for a micro-solenoid valve and particularly examines performance above the maximum rated frequency at design voltages. The results point positively toward the potential that a scaled-up version of this design could be capable of meeting the requirements of pulse detonation fuel injection and other high-speed applications.

\section{Valve Requirements}

For this study, four primary characteristics were considered in the valve selection process: (1) operating frequency, (2) response time, (3) service life and (4) valve architecture. The first two are interdependent since valves rated for high-frequency operation typically possess a fast response. Additional criteria included seals appropriate for use with hydrogen, gaseous hydrocarbons and oxygen, high working pressures (approximately 100 psig) and elevated temperatures.

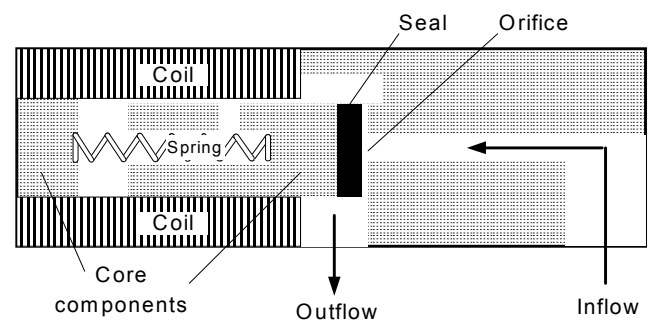

Figure 1. Cross-section of idealized solenoid valve.

A commercially available solenoid valve that partially fulfills the requirements stated above is one in the
Numatech ${ }^{\circledR}$ TM series, which has a maximum operating frequency of $50 \mathrm{~Hz}$, as quoted by the manufacturer. Other pertinent information from the manufacturer includes a response time of $3 \mathrm{~ms}$ for energizing and $5 \mathrm{~ms}$ for de-energizing. Power consumption is $1.2 \mathrm{~W}$ (or 1.35 $\mathrm{W}$, with LED indicator). This valve is a direct solenoid actuated, spring return, manifold mount, 3-way design available with Viton or Buna-N seals. The valve has a service life in excess of 200 million cycles and can operate at up to $690 \mathrm{kPa}$ (100 psig). Limitations include a limited working temperature range of $0-50{ }^{\circ} \mathrm{C}$, limited number of gaseous media and its small size. However, this valve possesses useful characteristics in that it can be dismantled and it can be easily modeled.

Depending on the configuration, the body is roughly $22 \times 27 \times 10 \mathrm{~mm}$. Fluid enters and exits the valve via three rectangular ports located side by side on the bottom of the valve, one inlet and two outlets. Each of the opening is $1 \mathrm{~mm} \times 3 \mathrm{~mm}$ square. Fluid, however, is injected into either of the two valve chambers through a $0.5 \mathrm{~mm}$ diameter circular cross-section orifice. Since the valve is a three-way design, there are two of these orifices.

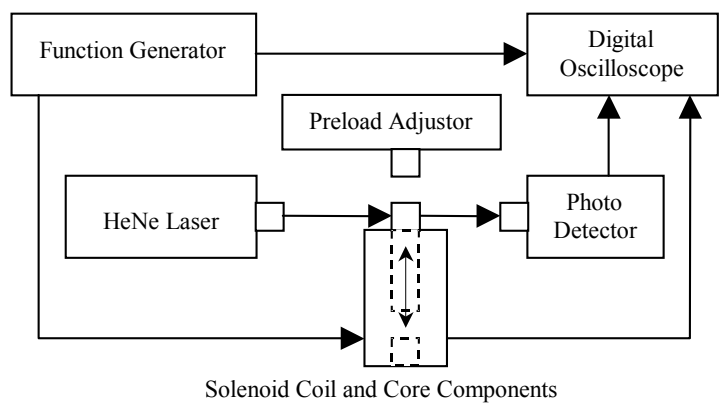

Figure 2. Schematic of experimental setup.

The valve design illustrated in Fig. 1 is particularly amenable to high-frequency applications and is comparatively easy to model. The movable portion of the design's split ferromagnetic core can be modeled as a simple mass-spring-damper system. A seal inserted in the end of the core blocks the injection orifice in the closed position and, when opened, allows the gas to expand rapidly into the chamber. A small vent through the core into the spring chamber serves as a mechanism to equalize the gas pressure as the valve chamber is filled. It is particularly advantageous since, as soon as the valve begins to open, the full orifice is available for injection. In rotary valves, on the other hand, the orifice opens and closes in phase, which may pose problems in controlling the flow.

\section{Experimental Methodology}

In order to observe the valve response independent of the valve casing, the solenoid coil and core components were 
separated from the valve assembly and secured in a brace to the original pre-loaded configuration. The solenoid coil and core components were tested using the setup illustrated schematically in Fig. 2.

A Metrologic (Blackwood, New Jersey) ML800 0.8 $\mathrm{mW}$ helium-neon laser was used to pass a confined beam of light perpendicular to the exposed movable core, the diameter of the beam being equivalent to the diameter of the core. A Thorlabs (Newton, New Jersey) Model DET200 high-speed photo detector (rise time of $1 \mathrm{~ns}$, spectral range of $200-1100 \mathrm{~nm}$ ) mounted on the opposite side measured the displacement response of the voltage-excited valve as a function of the time-varying light intensity. A BK Precision Model 3022 function generator supplied a square wave input signal of varying frequencies at approximately the manufacturer-rated input of 12 V. The input voltage, valve voltage, and photodetector output were recorded simultaneously by a HewlettPackard Model 5442A digital storage oscilloscope. A sampling rate of $25 \mathrm{kSa} / \mathrm{s} / \mathrm{channel}$ sufficiently resolved the response in the opening and closing phases at all tested frequencies. A maximum sample length of 16386 data points was collected during each test, corresponding to a sample duration of $0.655 \mathrm{~s}$. The oscilloscope trigger was set to initiate data collection five seconds into each test. The delay in data collection was chosen to allow the system sufficient time to stabilize.

\section{Modeling the Dynamic Response}

Most manifold mounted micro-valves are seated in the horizontal position, and (considering their small core masses) it is reasonable to neglect gravitational effects. Under this assumption the system dynamics can be represented by

$$
m \ddot{x}+C_{d} \dot{x}+F_{s}=F_{e}+F_{g}
$$

Unlike a free-response system, the motion of the core is constrained, and Eq. (1) is valid only over the allowed displacement range as determined by the valve geometry.

\section{Spring Constant}

At the full-open position, the spring is not completely compressed, but further displacement is limited by the fixed pole segment of the core. Assuming a linear relationship over the $0.5 \mathrm{~mm}$ displacement range, the spring force can be written as follows:

$$
F_{s}=k x+0.185
$$

with a pre-load force of $0.185 \mathrm{~N}$. Based on the manufacturer's data and using Eq. (2), the spring constant is 370 $\mathrm{N} / \mathrm{m}$. This value can be substantiated once the damping coefficient is determined as described in the following paragraphs.

\section{Mass and Damping Coefficient}

The combined mass of the movable core and spring were measured to be $0.605 \pm 0.002 \mathrm{~g}$ using an Acculab ${ }^{\mathrm{TM}} \mathrm{V}$ digital balance. The critical damping coefficient is given by $^{8}$

$$
C_{d, c r i t}=2 \sqrt{k m}=0.946 \mathrm{~kg} / \mathrm{s}
$$

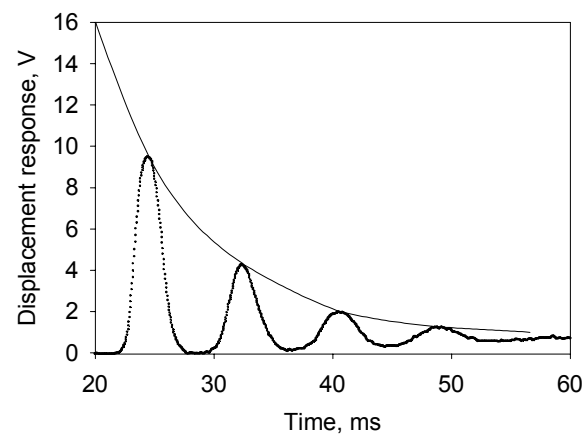

Figure 3. Undamped free response of valve core.

The logarithmic decrement describes the decay envelope of a free oscillating system. It is particularly amenable to the analysis of the damping ratio for simple mass/spring systems.

The free response to an initial displacement can be measured as a function of time by the method presented above. To do this, the spring is fixed to the core and the entire assembly is secured so that it is free to oscillate while partially constrained by the solenoid sleeve (see Fig. 1). An example of the data is shown in Fig. 3.

While the actual oscillation is sinusoidal, the negative portion of the signal shown in Fig. 3 is clipped since the valve core blocks the light beyond a $0.5 \mathrm{~mm}$ displacement. This is not an issue so long as the positive peaks are clearly defined and the true response is not constrained. The logarithmic decrement is then given by $^{8}$

$$
\delta=\frac{1}{n} \ln \left[\frac{x(t)}{x(t+n T)}\right]=0.6128
$$

using the data of Fig. 3. As the number of peaks counted increases, the accuracy of the method increases as well. The damping ratio for this system can be expressed in terms of the logarithmic decrement to yield

$$
\zeta=\delta / \sqrt{4 \pi^{2}+\delta^{2}}=0.0971
$$

while the damped natural frequency is $785.4 \mathrm{rad} / \mathrm{s}$. By definition, the natural frequency is

$$
\omega_{n}=\omega_{d} / \sqrt{1-\zeta^{2}}=\sqrt{k / m}=789 \mathrm{rad} / \mathrm{s}
$$

The spring constant was determined experimentally to be $377 \mathrm{~kg} / \mathrm{s}^{2}$. These experimental results for the natural 
frequency and spring constant are within 2 percent of that supplied by the manufacturer. The damping coefficient is given by

$$
C_{d}=2 \zeta \sqrt{\mathrm{km}}=\zeta C_{d, \text { crit }}=0.0927 \mathrm{~kg} / \mathrm{s}
$$

\section{Gas Pressure Force}

Micrometer measurements of the orifice diameter were $0.5 \mathrm{~mm}$. Similarly, micrometer measurements of the maximum core displacement - measured from the preloaded to full-open position-were also $0.5 \mathrm{~mm}$. At the maximum rated pressure of 100 psig, the gas pressure force through the orifice is approximately $0.136 \mathrm{~N}$. The manufacturer specified the spring preload force at 0.185 $\mathrm{N}$-sufficient to overcome this force-while the final load in the full-open position was stated at $0.370 \mathrm{~N}$. The small maximum displacement would do little to dissipate the pressure force exerted against the core tip, thus it should be sufficient in subsequent analyses to assume the gas pressure force to be constant. All experiments were conducted without flow. Thus the gas pressure term has been excluded from Eq. (1) in the analysis. The results of the previous discussion can now be used to rewrite Eq. (1) in a form that is representative of the actual experimental conditions and constraints:

$$
6.05 \times 10^{-4} \ddot{x}+9.27 \times 10^{-2} \dot{x}+376.75 x=F_{e}-0.185
$$

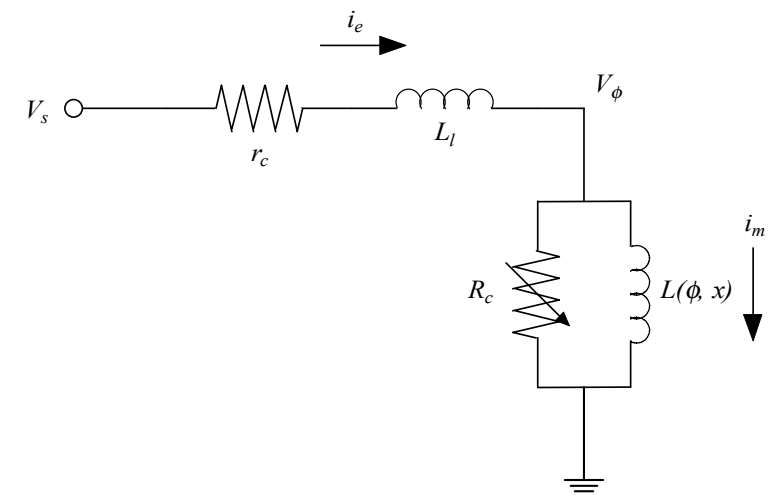

Figure 4. Solenoid valve equivalent circuit.

\section{Force Characteristics of a Non-linear Solenoid}

It is useful at this point to discuss the relationship between magnetic flux and electromagnetic force so as to more fully comprehend the nature of solenoid optimization. Figure 4 shows an equivalent circuit for a solenoid valve that helps to explain the nonlinear behavior of the valve's voltage and displacement response. The circuit elements include the actual coil resistance $r_{c}$, a variable flux leakage inductance $L_{l}$ (which is due to flux lines leaking through the air gaps and is responsible for shifting the flux versus current curve to the left or right), a non-linear coil inductance $L(\phi, x)$, and a variable core- loss resistance $R_{c}$ (which results from eddy current losses and determines the shape of the hysteresis loop about the steady-state magnetization curve-the larger its value, the wider the hysteresis loop). ${ }^{9}$

For a typical solenoid, there are two sources of core loss: eddy currents and hysteresis. Eddy currents circulating in the core material are the direct result of core voltages induced by the changing magnetic field. Hysteresis is the result of the energy dissipating properties of the core itself, acting as a variable resistance. The losses result in a difference between the exciting and magnetizing currents, $i_{e}$ and $i_{m}$, see equivalent circuit in Fig. 4. In other words, were there no losses, these values would be identical.

When current flows through a simple coil, magnetic flux is generated. The product of the magnetizing current and the number of coil turns is often referred to as the magnetomotive force (though not in the dimensional sense) and can similarly be expressed as the product of magnetic flux and total reluctance, ${ }^{9-11}$ or,

$$
i_{m}=\frac{\phi \cdot \Re}{N}
$$

The energy delivered to the coil can be expressed as the integral of power in terms of total reluctance and flux ${ }^{10}$

$$
W=\int_{0}^{\phi} N i_{m} \cdot d \phi=\int_{0}^{\phi} \Re \phi \cdot d \phi
$$

The electromagnetic force is expressed as the negative of the partial differential of the stored energy with respect to the variable air gap distance, the negative sign indicating the direction of the force, ${ }^{10}$

$$
F_{e}=-\frac{\partial w}{\partial x}=-\frac{\partial}{\partial x}\left(\int_{0}^{\phi} \Re \phi \cdot d \phi\right)
$$

using Eq. (10).

The total reluctance is equal to the sum of the component reluctances that include those from: the fixed air gap, the variable air gap, and the core material (see Appendix). ${ }^{11,12}$ Of these three terms, only the variable air gap contains the displacement $x$ so that Eq. (11) simplifies to

$$
F_{e}=-\frac{\partial}{\partial x}\left(\int_{0}^{\phi} \frac{x \phi}{\mu_{o} A_{x}} d \phi\right)=-\frac{\phi^{2}}{2 \mu_{o} A_{x}}
$$

The results clearly show the quadratic relationship between magnetic flux and electromagnetic force. At some point, the magnetic flux saturates. This point corresponds with the point of maximum force or minimum core loss. It is desirable for flux saturation to occur as quickly as possible thus maximizing the electromagnetic force and optimizing the opening phase of the solenoid valve. 


\section{Experimental Results}

The valve was tested at frequencies beginning with the manufacturer's stated maximum of $50 \mathrm{~Hz}$ up to $110 \mathrm{~Hz}$ in $10 \mathrm{~Hz}$ intervals and then again at $150 \mathrm{~Hz}$. At a sampling rate of $25 \mathrm{kSa} / \mathrm{s}$, the cycle accuracy for all tests was $40 \mu \mathrm{s}$. This corresponds to an accuracy of $\pm 0.2-0.6$ percent of the measured frequency at $50-150 \mathrm{~Hz}$. The sample length allowed approximately 33 consecutive cycles to be observed at $50 \mathrm{~Hz}$ and approximately 98 cycles at $150 \mathrm{~Hz}$. Thirty cycles from each data set were used to construct averaged response plots.

A set of sample results is shown in Fig. 5 for a 50 $\mathrm{Hz}$ duty cycle with the displacement response scaled to the input signal. Figure 5a shows a sample of the data. The excellent repeatability is demonstrated in Fig. 5b by overlaying eight consecutive cycles. The average of thirty consecutive cycles is shown enlarged in Fig. 5c. In Figs. $5 \mathrm{~b}$ and $\mathrm{c}$, the input and solenoid output voltages are

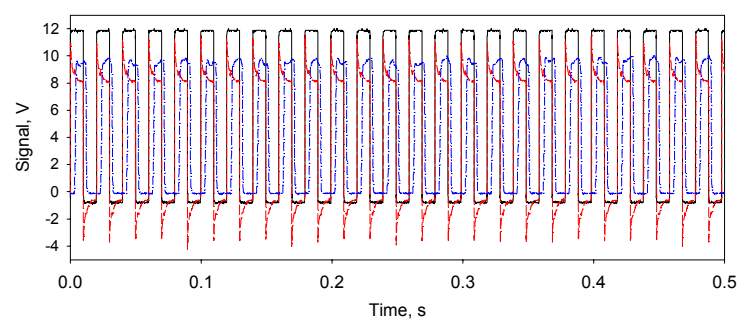

a. Data. normalized by the averaged, measured input value while the photodetector output is normalized by the maximum photodetector output.

The $12 \mathrm{~V}$, square-wave excitation shows a slight negative offset. The solenoid output immediately follows the excitation. Motion of the core begins when the magnetic field strength is sufficient to overcome the spring force and draw the core into the field. Initially the boss end extends slightly beyond the field influence and eddy currents are low. However, as the core moves more fully into the field, eddy currents increase resulting in a corresponding temporary decrease in the magnetizing current. As this occurs, the solenoid force is weakened, resulting in a temporary slowdown in core displacement as seen by the slight reduction in the slope of the displacement curve, Fig. 5c. Once flux saturation occurs, the core loss resistance stabilizes, the flux leakage inductance decreases which increases the magnetizing current, and, consequently, the electromagnetic force and rate of

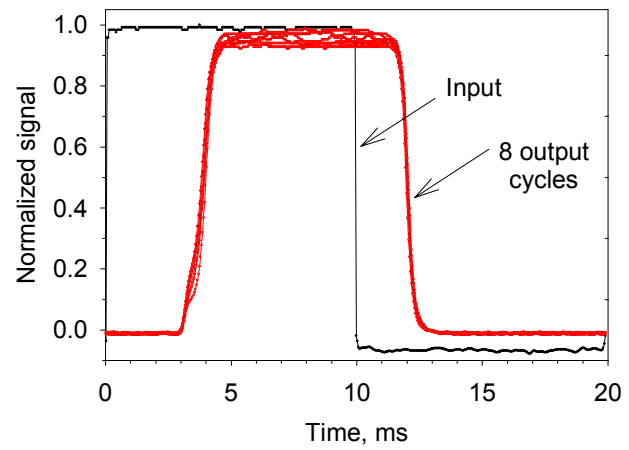

b. Repeatability check - eight consecutive cycles.

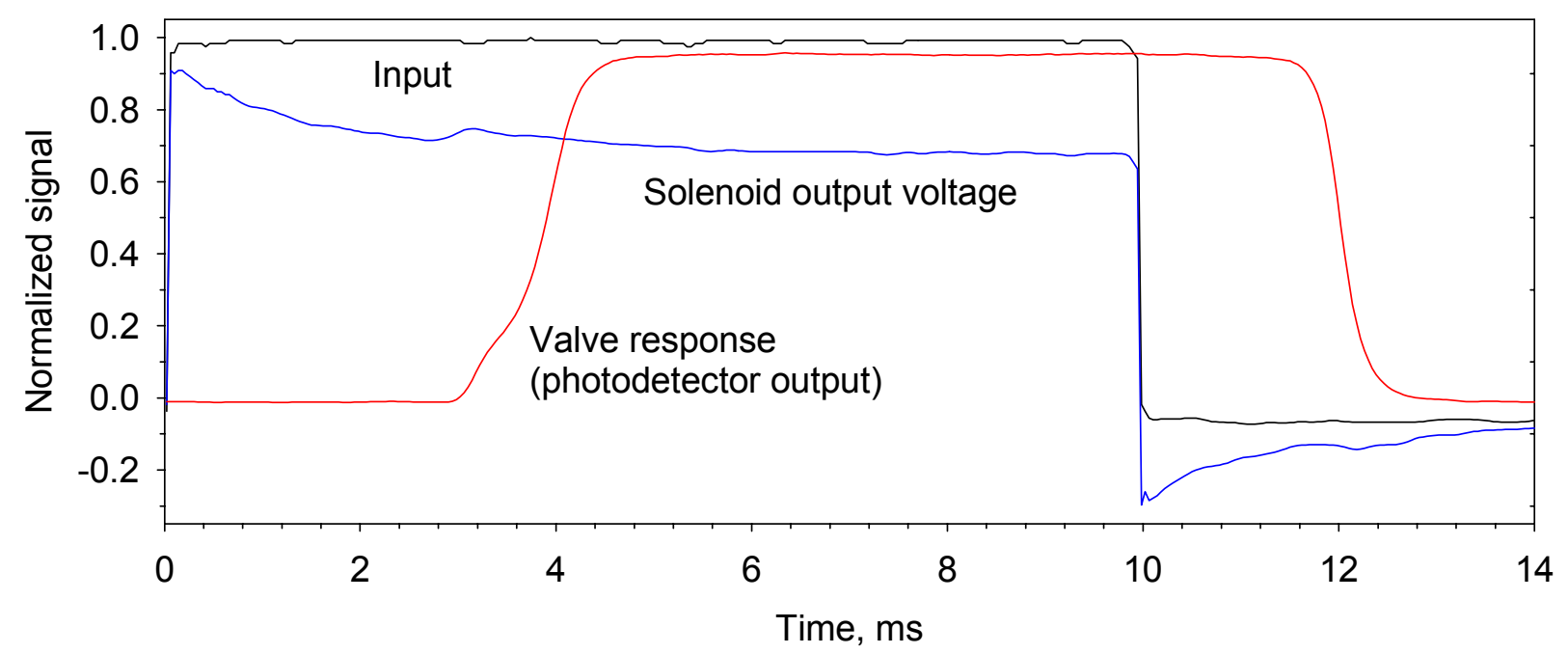

c. Average of eight cycles.

Figure 5. Input and outputs at $50 \mathrm{~Hz}$ excitation. 


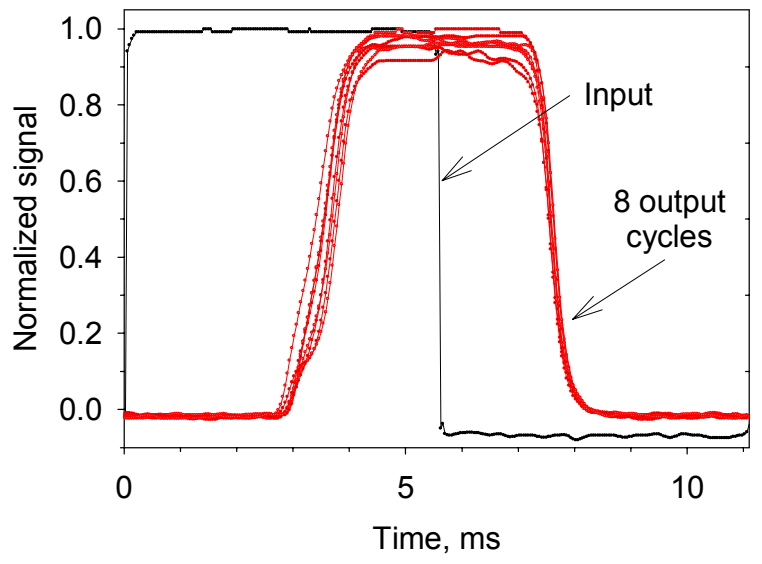

Figure 6. Repeatability check at $90 \mathrm{~Hz}-$ eight consecutive cycles.

displacement. The rise in voltage observed just prior to the opening displacement phase is the result of an induced voltage created by the changing magnetic field. While still present, these effects are less pronounced during the closing phase as the magnetic field collapses.

The phenomena described above are observed in all data samples and at all frequencies tested. For example, Figs. 6 and 7 show the normalized output of eight cycles at 90 and $100 \mathrm{~Hz}$ operation. Some deterioration in opening phase repeatability is evident at $100 \mathrm{~Hz}$, and significant cycle deterioration is evident at $150 \mathrm{~Hz}$, the highest frequency tested (Fig. 8).

The opening and closing response times of the valve at $50-110 \mathrm{~Hz}$ are shown in Fig. 9. It is important to keep in mind that the geometry of the valve tested (Fig. 1) possesses a significant advantage over other valve types, specifically rotary types - this being that there is no partial open or closed position. The slightest motion of the core breaks the seal, allowing the immediate inflow of gas. While the data illustrates a finite time to fully open or close the valve, it is expected that the mass flow will

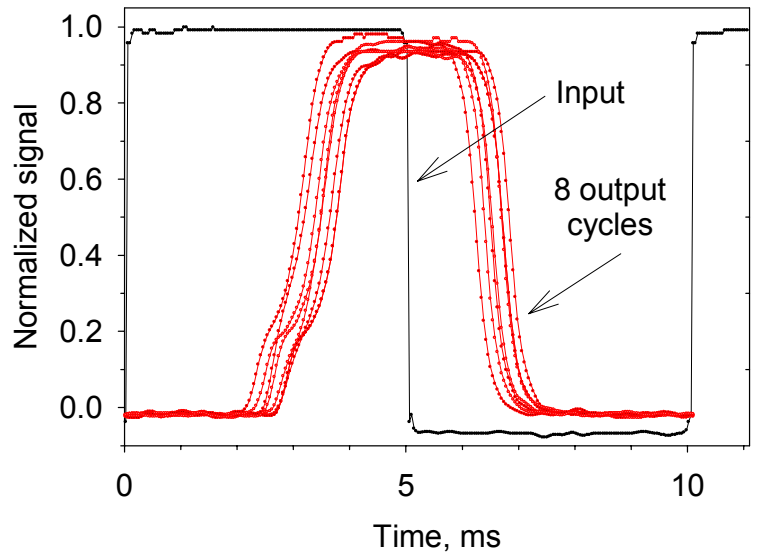

Figure 7. Repeatability check at $100 \mathrm{~Hz}$ - eight consecutive cycles.

closely match the input signal characteristics rather than the core response, being primarily governed by gasdynamic considerations. If this is indeed the case, whether or not the valve fully opens will not be as critical as the meticulous control of the cycle time as a means of delivering a precise gaseous quantity. Thus - considering the desired $0.3 \mathrm{~ms}$ response time as stated previously-it is not unreasonable to expect that maximum gas flow might be achieved when the valve is only partially retracted. Figure 9 shows that the rise and fall times do not vary with frequency but remain virtually constant, while the increasing phase lag tends to diminish the full open time. These observations confirmed unpublished analysis.

Based on the data in Fig. 9, the combined averaged time from initial excitation to full displacement is approximately $8.6 \mathrm{~ms}$, implying that, at any frequency above $116 \mathrm{~Hz}$, the input signal will be terminated prior to the valve being completely open. Thus, in order for valid tests to be conducted above this frequency, the duty cycle of the input signal would have to be increased. For example, this can be seen by the obvious cycle failures in

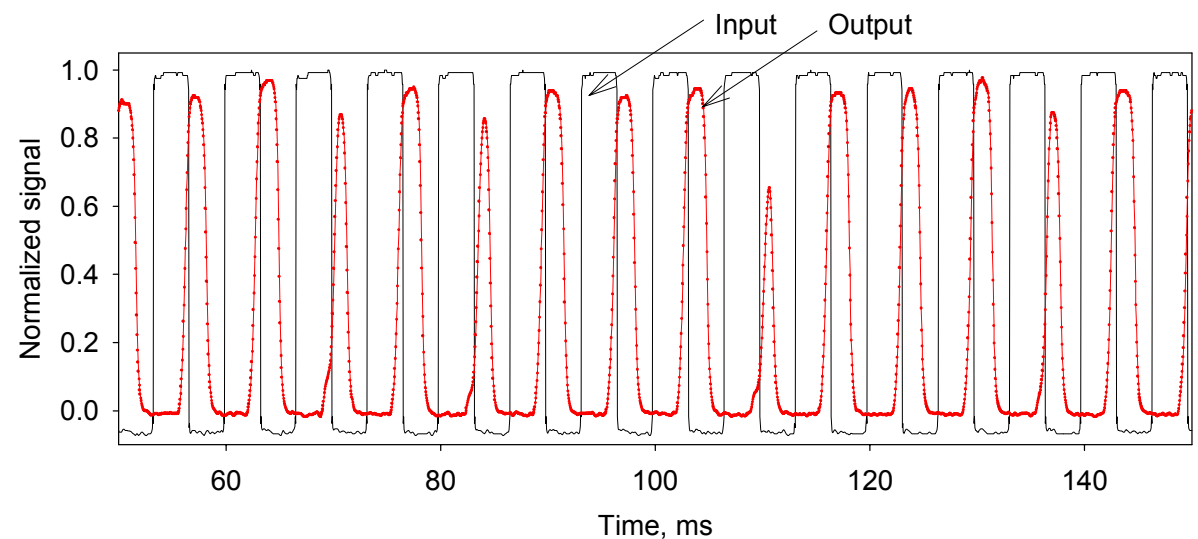

Figure 8. $150 \mathrm{~Hz}$. 
Fig. 8.

Figure 9 illustrates an unexpected result. Over the range of tested frequencies, the valve's dynamic performance at the recommended input voltage appears optimized at $70-90 \mathrm{~Hz}$, or 140 to 180 percent above the manufacturer's stated maximum frequency (without consideration given to valve longevity). When combined with the results of repeatability, the range of $70-80 \mathrm{~Hz}$ is clearly optimal. These results may be due to hysteresis effects or differences in the loss mechanisms caused by the higher frequency input so long as the maximum frequency for the input signal duty cycle is not exceeded. A more detailed examination of the phenomena is required before definite conclusions can be drawn. Nevertheless, the results point encouragingly toward the capability of simple spring-return solenoid devices to function at the frequency levels demanded by pulse detonation applications, consistently and reliably.

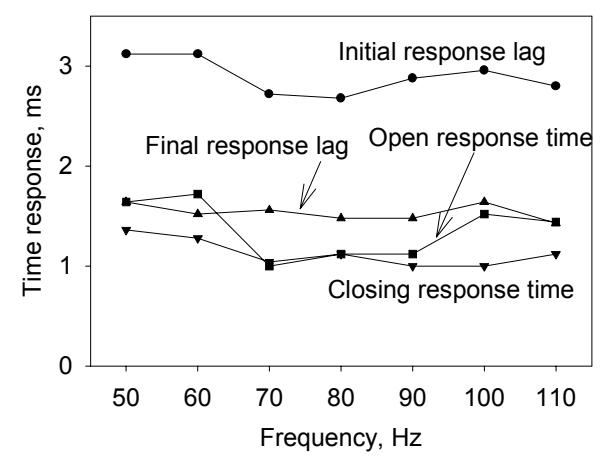

Figure 9. Response characteristics.

\section{Conclusions}

A miniature solenoid valve was tested under no-load conditions. The architecture of this particular valve type facilitated the understanding of valve operation at high frequency. It was found that the valve opening lagged by a constant time delay for a range of frequencies from 50 $110 \mathrm{~Hz}$ when excited at $12 \mathrm{~V}$. The motion of the solenoid core could be understood in terms of the interaction between the electromagnetic and spring forces. The magnetizing current lags the exciting current creating a phase delay at all frequencies. When the cycle frequency increases, however, the cycle-to-cycle repeatability deteriorates and the solenoid's behavior became erratic at $150 \mathrm{~Hz}$.

\section{Acknowledgements}

D. Jensen was supported by a McNair-SOAR scholarship while performing this research. Additional support was made available by the State of Texas Advanced Technology Program grant No. 003656-0198.

\section{References}

1. Bussing, T.R.A., and Pappas, G., "An Introduction to Pulse Detonation Engines," AIAA Paper 94-0263, Jan. 1994.

2. Bussing, T.R.A., and Pappas, G., "Pulse Detonation Engine Theory and Concepts," Developments in High-Speed Vehicle Propulsion Systems, Progress in Astronautics and Aeronautics, AIAA, Reston, VA Vol. 165, 1996, pp. 421-472.

3. Kawai, S. and Fujiwara, T., "Numerical Analysis of First and Second Cycles of Oxyhydrogen PDE," AIAA Paper 2002-0929, 2002.

4. Ohdachi, Y., Kawase, Y., Murakami, Y., and Inaguma, Y., "Optimum Design of Dynamic Response in Automotive Solenoid Valve," IEEE Transactions on Magnetics, Vol. 27, No. 6, 1991, pp. 5226-5228.

5. Hong, H., Krepec, T., Cheng, R. M. H., "Transient Response of Fast Acting Solenoids in Automotive Applications," Journal of Circuits, Systems, and Computers, Vol. 4, No. 4, 1994, pp. 415-428.

6. Hong, H., "Optimum Performance of Solenoid Injectors for Direct Injection of Gaseous Fuels in IC Engines," Ph.D. Dissertation. Mechanical Engineering Department, Concordia University, Montreal, Quebec, Canada, 1995.

7. Mitsutake, Y. and Hirata, K., "Dynamic Response Analysis of a Linear Solenoid Actuator," IEEE Transactions on Magnetics, Vol. 33, No. 2, 1997, pp. 1634-1637.

8. Inman, D.P., Engineering Vibration, $2^{\text {nd }}$ ed., Prentice Hall, Englewood Cliffs, New Jersey, 2001.

9. Hambley, A.R., Electrical Engineering-Principles and Applications, Prentice Hall, Englewood Cliffs, New Jersey, 1997.

10. Kraus, J.D., Electromagnetics, McGraw Hill, New York, 1953.

11. Hunt, W.T. and Stein, R., Static Electromagnetic Devices, Allyn and Bacon, Boston, 1963.

12. Coren R. L., Basic Engineering Electromagnetics, Prentice Hall, Englewood Cliffs, New Jersey, 1989.

\section{Appendix: Development of Solenoid Reluctance}

Theoretically, if the material permeability can be assumed constant, the reluctance is equal to the length of the path in the direction of the magnetic flux divided by the product of the permeability and the effective area normal to the path. ${ }^{10,11}$ For most solenoids, three reluctances must be considered: the fixed air gap reluctance, the variable core air gap reluctance, and the core material reluctance. The first two involve air whose permeability can be assumed constant, and the standard equation can be used. The reluctance of the fixed air gap is then

$$
\Re_{g}=\frac{g_{f}}{\mu_{o} A_{g}}
$$


where $A_{g}$ and $g_{f}$ are the fixed air gap cross-sectional area and the fixed air gap length, respectively, both in the direction of the magnetic flux path. The reluctance of the variable core air gap is

$$
\Re_{x}=\frac{x}{\mu_{o} A_{x}}
$$

where $A_{x}$ and $x$ are the variable air gap cross-sectional area and the variable air gap length, respectively, both in the direction of the magnetic flux path. The core reluctance can be expressed as

$$
\Re_{m}=\frac{f_{2}}{f_{1}-\phi}
$$

where $f_{1}$ and $f_{2}$ are constants related to the magnetization characteristics of ferromagnetic materials. ${ }^{6}$

From the definition of the magnetomotive force, the magnetizing current in a simple coil can be expressed in terms of the product of flux and total reluctance divided by the number of coil turns,

$i_{m}=\Re_{g}+\Re_{x}+\Re_{m}=\frac{\phi}{N} \cdot\left(\frac{g_{f}}{\mu_{o} A_{g}}+\frac{x}{\mu_{o} A_{x}}+\frac{f_{2}}{f_{1}-\phi}\right)$

which is equivalent to Eq. (9) in the main text. 